\title{
Validation of Presumptive Test for Non-Human Blood using Kastle Meyer and Hemastix Reagents
}

Casali, $\mathrm{F}^{\mathrm{a}, \mathrm{b}}$, Ciavaglia, $\mathrm{SA}^{\mathrm{b}}$, Gannicliffe, $\mathrm{C}^{\mathrm{c}}$, Lidstone, $\mathrm{N}^{\mathrm{a}, \mathrm{d}}$, and LMI Webster ${ }^{\mathrm{b}}$
a. Centre for Forensic Science, Department of Pure and Applied Chemistry, University of Strathclyde
b. Wildlife DNA Forensics Unit, SASA, Edinburgh, EH12 9FJ, United Kingdom
c. Forensic Services, Scottish Police Authority
d. University of Staffordshire
Corresponding author:
Lucy M I Webster
lucy.webster@sasa.gov.scot

\section{Declarations of interest: none}




\section{Abstract}

Kastle Meyer and Hemastix reagents are blood presumptive tests commonly used in forensic casework for the detection of human blood, and their suitability have been reviewed by numerous publications. However until now, no published work has looked at the sensitivity, specificity and effect on DNA when using these reagents to presumptively test for animal blood. The aim of this study was to validate the two reagents for use with animal blood, and to compare their performance in order to choose the best test based on the circumstances in wildlife crime investigation.

The sensitivity, specificity, stability and robustness of the methods were assessed by experiments with dilutions of blood (from 1:4 to 1:65596) using direct and indirect (rub) tests, potential interfering substances, blood sources from different species and aged blood. Furthermore, interference of the two reagents on subsequent DNA analysis was investigated.

During the direct tests, Kastle Meyer showed a higher sensitivity, detecting blood down to a dilution of 1:16384, one order of magnitude more than Hemastix. However during the rub test, Hemastix showed a higher sensitivity, detecting blood down to a dilution of 1:64 on porous materials while Kastle Meyer was positive only down to a dilution of 1:16. Moreover, when using the same swab for presumptive testing and DNA extraction, Hemastix testing allowed amplification of a sufficient amount of DNA for species identification down to a dilution of 1:1024 while Kastle Meyer inhibited amplification of DNA at a 1:16 dilution. On the other hand, Hemastix showed a much lower specificity, producing false positive results when exposed to tomato, potato, rust, avian uric acid, bleach and sink rot, while Kastle Meyer only produced a faint positive reaction from potato. Both tests performed equally well detecting fresh blood of different animal species. Finally, both tests showed comparable results during the stability test, but Kastle Meyer was not able to detect aged-fish blood stains in the majority of cases.

Owing to its ease of use, higher sensitivity, and lack of interference with downstream DNA analysis, and despite its reduced specificity compared to Kastle Meyer, the Hemastix method is more appropriate for use in wildlife crime investigations. Positive results would always be confirmed with DNA analysis and the low interference of the reagent will allow the use of a single swab for presumptive testing and DNA sampling.

Keywords: wildlife forensic science; presumptive blood testing; Kastle Meyer; Hemastix; nonhuman; DNA 


\section{Introduction}

Blood presumptive tests, such as the Kastle-Meyer (KM) test and Hemastix, are commonly used in forensic casework to determine the possible presence of blood if, for example, a red-brownish stain is found on a garment. These tests do not give an examiner full confidence that the stain is blood, because various other substances can react with reagents used in these tests to give false positive results, for example tomato and red onion [1]. To be able to correctly identify a stain as being produced by blood, a further confirmatory test is needed, such as Teichmann's and Takayama's tests [2] [3], albeit these are not generally in widespread use in forensic casework. The negative result of a presumptive test can suggest an absence of haemoglobin on the object of interest, or presence of haemoglobin at a concentration below the sensitivity of the test. However, it must be also noted that false negative results can arise in presence of various anti-oxidant substances, such as ascorbic acid (Vitamin C) [4].

Another issue with the use of presumptive tests for blood is the wide range of sensitivity recorded in literature: for the KM test, different authors have found dramatically different thresholds of sensitivity in dilutions of blood, spanning from 1:10,000 [1], 1:100,000 [5], 1:1,000,000 [6] to 1:100,000,000 [4]. A similar variation was also found during the sensitivity testing of Hemastix [1] $[5]$.

While it is expected that presumptive blood tests will work on animal blood as well as human blood, no validation studies have been published to formally assess this. Both presumptive tests chosen for this study, KM and Hemastix, use the peroxidase activity of the heme group of haemoglobin as a catalyst that oxidises in the presence of hydrogen peroxide [7].

Wildlife crimes often involve the unlawful killing of animal species, and DNA analysis is routinely used in wildlife forensic casework to identify species from blood stains [9]. The potential interference of KM or Hemastix reagents with subsequent DNA analysis during direct testing is a subject of interest [10] [11] [12]. Human DNA has been successfully recovered from blood stains treated with both KM and Hemastix, although the former caused a larger reduction in the amount of DNA recovered [1]. Hemastix has been found to prevent recovery of DNA using the Promega DNA IQ System extraction technique, probably due to the binding of the dye (tetramethylbenzidine) to the magnetic beads [10]. The KM reagent has also been shown to be responsible for the degradation of DNA contained in minute blood stains, and that degradation increased with time of exposure to the reagent before DNA extraction [11]. Finally, after direct testing, the sodium hydroxide content of KM reagent can significantly reduce the quantity of detectable human DNA [12]. 
The aim of this study is to validate the use of KM and Hemastix presumptive tests in wildlife crime investigations involving a range of species and to highlight any differences between the two tests. In addition, the study will examine possible interference of KM and Hemastix reagents with subsequent animal DNA analysis. This will demonstrate the suitability of presumptive testing for animal blood, identify the most suitable method for the use in forensic wildlife casework and potentially streamline the testing process if directly tested swabs can be used for DNA analysis.

\section{Materials \& Methods}

\subsection{Samples}

Blood from various animal species were sourced for this project. Dog blood was obtained as a biproduct of a veterinary procedure, salmon blood was obtained from a fish supplier and chicken, horse, sheep, rabbit and guinea pig blood was purchased from TCS Bioscience. Chicken, dog and salmon blood were used as representatives of bird, non-human mammal and fish groups to perform sensitivity and stability tests. Horse blood was used as a positive control of the reagents and for the specificity test [13].

\subsection{Reagents and presumptive test method}

The KM presumptive blood testing kit (Scenesafe) and Hemastix test strips (Siemens) were used following the manufacturer's guidelines. For KM, standard operating procedures from the Scottish Police Authority were followed. In brief for KM, phenolphthalin was added to the swab used to sample a stain. Provided no colour change was observed after ten seconds, hydrogen peroxide was added to the swab. A positive result was recorded if a colour change towards pink arose within 30 seconds. For Hemastix, the stained swab was applied to the testing pad and a positive result was recorded if a colour change of the pad towards green/blue arose within 60 seconds. Positive controls were performed by applying the reagents to a horse blood-stained swab. Negative controls were performed by applying the reagents to a distilled water moistened, unstained swab.

\subsection{Sensitivity}

Dilutions were created for chicken, dog and salmon blood after initial standardisation from the supplier concentration using DNA-free water. Each undiluted blood sample was used to create five independent dilution series for each species, using a serial dilution factor of four. The tested concentrations were: 1:4, 1:16, 1:64, 1:256, 1:1024, 1:4096, 1:16384 and 1:65536. During preparation, each dilution was thoroughly mixed by hand and by pipetting to produce a homogeneous solution. 
The first round of sensitivity experiments employed direct testing and rub testing. Firstly, for the direct test, blood dilutions were spotted twice (once for each presumptive method) adding $5 \mu \mathrm{L}$ of each dilution onto single squares of filter paper $\left[2 \mathrm{~cm}^{2}\right]$. The stains were left to dry overnight in a fume hood. The presumptive test reagents were then applied directly to the prepared stains.

The rub test, referred to as the rub test, involved producing stains by placing $5 \mu \mathrm{L}$ of each blood dilution on filter paper strips $[7 \mathrm{~cm} * 3 \mathrm{~cm}]$ at an appropriate distance so to prevent crosscontamination. Five strips were prepared for each dilution factor. These stains were then rubbed with a moistened swab, to which the reagents from the two tests were subsequently added.

All spots were colourless after 1:64 dilutions, and so for weaker dilutions the outer edge of the stain was marked with pen while still wet.

A second round of sensitivity experiments was performed using only the rub test method with a narrower set of dilutions to assess sensitivity on different surface types. Stains were spotted on two different substrates: non-stainless steel and fabric were chosen to mimic items commonly present in wildlife crime scenes, such as a metal animal trap or a piece of cloth from a shirt.

As for the first experiment, five replicas of each dilution for the three blood types were used. Ten microliters of each dilution was added onto the two different surfaces, fabric strips [12cm * $13 \mathrm{~cm}]$ and metal trap part $[55 \mathrm{~cm} * 40 \mathrm{~cm}]$, distancing the spots to avoid cross-contamination.

The different bloodstains were randomised within a single fabric strip and between dilutions on metal. The randomisation was performed to account for potential variability across the material.

\subsection{Specificity}

Two specificity experiments were performed. The first examined a variety of substances that could potentially create false positive and false negative results from the test, and the second investigated specificity to blood from a variety of animal species.

A number of substances previously reported to produce false positive results with the two reagents were studied [1] using the rub test method. Other substances common to wildlife crime scene environments were included. The samples used were: tomato, potato, rust, chainsaw oil, bleach, sun cream, WD40 lubricant, petrol, grease, gun oil, avian uric acid, sink rot, EDTA, Alsever's solution and Chemgene disinfectant.

These substances were first tested alone to record any false positive results using both KM and Hemastix. Each substance was used to stain a swab, which was then treated with the respective 
reagents. The substances were subsequently mixed with horse blood and tested with both reagents, to see if the combination of the two substances would produce false negative results. Five microlitres of horse blood was added to the swabs stained with each substance. All tests were performed in five replicates.

The species specificity experiment used blood from dog, salmon, chicken, horse, sheep, rabbit and guinea pig to record any difference in reactivity of the KM and Hemastix tests. Five replicate tests were performed.

\subsection{Stability}

Bird, mammal and fish blood were used to stain four strips of fabric [10 $\mathrm{cm} * 6 \mathrm{~cm}]$. Prior to staining, the strips were decontaminated using a commercial household laundry detergent and then irradiated on both sides with UV light to eliminate exogenous DNA. Five neat bloodstains (5-10 $\mu \mathrm{L}$ ) of each species were spotted per strip, separated by a distance of $\sim 1 \mathrm{~cm}$. The different bloodstains were randomised within a single strip to account for potential variability across the fabric. The same randomised bloodstains positions were used on all four fabric strips. The first strip ( $\underline{\text { control) }}$ was left to rest for five weeks inside a cabinet in complete darkness. The second strip

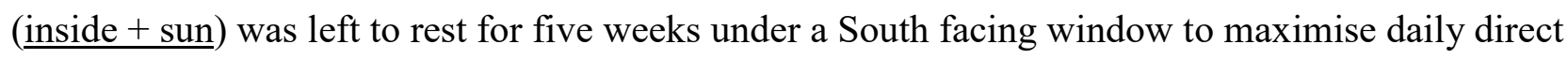
sunlight exposure. The third strip (outside + cover) was left to rest for five weeks inside a wooden

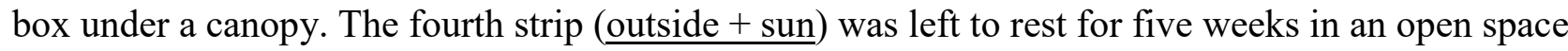
with exposure to the South to receive the highest amount of sunlight each day; this strip was affected by all environmental conditions. At the end of the five week period, the four sets were tested with both KM and Hemastix using the rub test method. Three replicates tests were performed on each bloodstain with each respective reagent.

\subsection{Effect on downstream DNA analysis}

Swab samples from the initial sensitivity study were chosen for all three species chosen for this work, based on the lowest dilution to yield positive results from KM or Hemastix reagents.

Qiagen QIAamp DNA investigator kit was used to perform DNA extraction following the manufacturers' protocol.

After extraction, DNA was quantified using a NanoDrop spectrophotometer prior to PCR amplification. Each PCR mix included 1x Type-IT mastermix (Qiagen), $0.5 \mu \mathrm{M}$ each primer, $2 \mu 1$ DNA extract made up to a final volume of $20 \mu \mathrm{l}$. Based on the species-origin of the blood, different methods were employed that would target DNA in the species of interest. For swabs from chicken 
blood, primers Chicken_COIF (5'-GCCGGCACAGCACTTAGCCT-3') and Chicken_COIR (5'GGTCTCCTCCTCCAGCTGGGTC-3') were used [16] under the following cycling conditions: 5 $\min 95^{\circ} \mathrm{C},\left(95^{\circ} \mathrm{C} 30 \mathrm{~s}, 65^{\circ} \mathrm{C} 30 \mathrm{~s}\left(-0.5^{\circ} \mathrm{C}\right.\right.$ per cycle $\left.), 72^{\circ} \mathrm{C} 30 \mathrm{~s}\right) \times 10$ cycles, $\left(95^{\circ} \mathrm{C} 30 \mathrm{~s}, 60^{\circ} \mathrm{C} 30 \mathrm{~s}, 72^{\circ} \mathrm{C}\right.$ $30 \mathrm{~s}) \times 25$ cycles,, $72^{\circ} \mathrm{C} 5 \mathrm{~min}$ and a final hold at $15^{\circ} \mathrm{C}$. For swabs from dog blood, primers D2 (5'CTCCCTAAGACTCAAGGAAGAAGC-3') and D5 (5'- CCCTAAAACTATATGTCCTGAA-3') were used [17], with the following cycling conditions: $5 \min 95^{\circ} \mathrm{C},\left(95^{\circ} \mathrm{C} 30 \mathrm{~s}, 52^{\circ} \mathrm{C} 60 \mathrm{~s}, 72^{\circ} \mathrm{C} 60 \mathrm{~s}\right)$ x 35 cycles, $72^{\circ} \mathrm{C} 5 \mathrm{~min}$, and a final hold at $15^{\circ} \mathrm{C}$. Finally for swabs from salmon blood, primers Ssal_COI_163F(5'-CGACATAGCATTCCCCCGAA-3') and Ssal_COI_506R (5'CTGCTGCTAGAACAGGGAGG-3') were used [16] under the following cycling conditions: 5 $\min 95^{\circ} \mathrm{C},\left(95^{\circ} \mathrm{C} 30 \mathrm{~s}, 65^{\circ} \mathrm{C} 30 \mathrm{~s}\left(-1^{\circ} \mathrm{C}\right.\right.$ per cycle $\left.), 72^{\circ} \mathrm{C} 30 \mathrm{~s}\right) \times 8$ cycles, $\left(95^{\circ} \mathrm{C} 30 \mathrm{~s}, 58^{\circ} \mathrm{C} 30 \mathrm{~s}, 72^{\circ} \mathrm{C} 30 \mathrm{~s}\right)$ x 27 cycles, $72^{\circ} \mathrm{C} 5 \mathrm{~min}$, and a final hold at $15^{\circ} \mathrm{C}$.

Following the PCR reaction, $5 \mu \mathrm{l}$ of product was run on an agarose gel to check for successful amplification.

\subsection{Statistical tests}

The test results were nominal variables: a reaction was either present or absent. Fisher's exact test of independence was therefore used to test whether the proportion of one variable (e.g. results of KM with bird blood) was different depending on the value of the other variable (e.g. results of Hemastix with bird blood). Fisher's exact test is more accurate than the Chi-square or G-test of independence when the expected numbers are small [13]. 


\section{Results}

\subsection{Sensitivity}

For the first sensitivity experiment using direct testing, all replicates for each species gave positive results using both $\mathrm{KM}$ and Hemastix tests at dilutions 1:4, 1:16, 1:64 and 1:256. The KM reagent gave positive results across the entire range of dilutions for mammal and fish blood. For bird blood, positive results with $\mathrm{KM}$ were obtained down to 1:256, with the exception of a single replicate result at 1:1024 (Table 1).

Table 1 - Subset of first sensitivity experiment using direct testing - all stronger dilutions were consistently positive. Presence of reaction $(+)$ is shaded in grey and absence (-) unshaded. B = bird; $\mathrm{M}=$ mammal; $\mathrm{F}=$ fish.

\begin{tabular}{|c|c|c|c|c|c|c|c|}
\hline \multirow{3}{*}{ Dilutions } & \multirow{3}{*}{ Replicates } & \multicolumn{6}{|c|}{ DIRECT TESTING } \\
\hline & & \multicolumn{3}{|c|}{ KM } & \multicolumn{3}{|c|}{ HEMASTIX } \\
\hline & & B & $\mathrm{M}$ & $\mathrm{F}$ & B & $\bar{M}$ & $\mathrm{~F}$ \\
\hline \multirow{5}{*}{$1: 256$} & 1 & + & + & + & + & + & + \\
\hline & 2 & + & + & + & + & + & + \\
\hline & 3 & + & + & + & + & + & + \\
\hline & 4 & + & + & + & + & + & + \\
\hline & 5 & + & + & + & + & + & + \\
\hline \multirow{5}{*}{$1: 1024$} & 1 & - & + & - & + & + & + \\
\hline & 2 & - & + & + & + & + & + \\
\hline & 3 & + & + & + & + & + & - \\
\hline & 4 & - & + & + & + & + & - \\
\hline & 5 & - & + & + & - & + & + \\
\hline \multirow{5}{*}{ 1:4096 } & 1 & - & + & + & - & + & - \\
\hline & 2 & - & + & + & - & + & - \\
\hline & 3 & - & + & + & - & + & - \\
\hline & 4 & - & + & + & - & + & - \\
\hline & 5 & - & + & + & - & + & - \\
\hline \multirow{5}{*}{$1: 16384$} & 1 & - & + & + & - & - & - \\
\hline & 2 & - & + & + & - & - & - \\
\hline & 3 & - & + & + & - & - & - \\
\hline & 4 & - & + & + & - & - & - \\
\hline & 5 & - & + & + & - & - & - \\
\hline
\end{tabular}

At dilution 1:1024, bird blood treated with $\mathrm{KM}$ gave a positive response $20 \%$ of the time, as opposed to $100 \%$ from mammal and $80 \%$ from fish blood. A significant difference in responses was found between bird and mammal blood $(p=0,048)$, but not between other comparisons. After dilution 1:4096, significant difference in responses with KM was found between bird blood, which 
gave no positive response, and the other two species that reacted positively at both concentrations ( $p$ $=0,008)$.

The Hemastix test showed reduced sensitivity compared to the KM when direct testing (Table 1). Positive results were recorded down to a dilution of 1:1024 for bird and fish, and 1:4096 for mammal blood. Significant difference in responses was found between mammal blood and the other two species $(\mathrm{p}=0,008)$.

The rub test, or indirect test, results were much less sensitive than the direct tests (Table 2). Positive results for KM occurred down to a dilution of 1:16, while positive reaction with Hemastix were seen down to a dilution of 1:64. All weaker dilutions were consistently negative.

Table 2 - Subset of first sensitivity experiment using rub testing. All weaker dilutions were consistently negative. Presence of reaction $(+)$ is shaded in grey and absence $(-)$ unshaded. B = bird; $\mathrm{M}=$ mammal; $\mathrm{F}=$ fish.

\begin{tabular}{|c|c|c|c|c|c|c|c|}
\hline \multirow{3}{*}{ Dilutions } & \multirow{3}{*}{ Replicates } & \multicolumn{6}{|c|}{ RUB TEST } \\
\hline & & \multicolumn{3}{|c|}{ KM } & \multicolumn{3}{|c|}{ HEMASTIX } \\
\hline & & B & $\mathrm{M}$ & $\mathrm{F}$ & B & $\mathrm{M}$ & $\mathrm{F}$ \\
\hline \multirow{5}{*}{$1: 4$} & 1 & + & + & + & + & + & + \\
\hline & 2 & + & + & + & + & + & + \\
\hline & 3 & + & + & + & + & + & + \\
\hline & 4 & + & + & + & + & + & + \\
\hline & 5 & + & + & + & + & + & + \\
\hline \multirow{5}{*}{$1: 16$} & 1 & + & + & + & + & + & + \\
\hline & 2 & + & + & + & + & + & + \\
\hline & 3 & + & + & + & + & + & + \\
\hline & 4 & + & + & + & + & + & + \\
\hline & 5 & + & + & + & + & + & + \\
\hline \multirow{5}{*}{$1: 64$} & 1 & - & - & - & + & + & + \\
\hline & 2 & - & - & - & + & + & + \\
\hline & 3 & - & - & - & + & + & + \\
\hline & 4 & - & - & - & + & + & + \\
\hline & 5 & - & - & - & + & + & + \\
\hline
\end{tabular}

No significant difference was found for either reagent among the three species. From all three species, KM produced $100 \%$ positive responses at dilutions $1: 4$ and 1:16, and no positive responses at dilution 1:64. Hemastix produced 100\% positive responses for all three dilutions with all blood types. Significant differences were found for all of the three species $(p=0,008)$ at dilution 1:64. 
Following the results of the first sensitivity study with the rub test, dilutions 1:4, 1:16 and 1:64 were selected to investigate variations in sensitivity based on surface type. The results of the rub testing on fabric were in line with the rub test results obtained in the first sensitivity study (Table 3 ).

Table 3 - Results from rub test experiment. Presence of reaction $(+)$ is shaded in grey and absence (-) unshaded. $\mathrm{B}=$ bird; $\mathrm{M}=$ mammal; $\mathrm{F}=$ fish.

\begin{tabular}{|c|c|c|c|c|c|c|c|c|c|c|c|c|c|}
\hline \multirow[b]{3}{*}{ Dilutions } & \multirow[b]{3}{*}{ Replicates } & \multicolumn{6}{|c|}{ FABRIC } & \multicolumn{6}{|c|}{ METAL } \\
\hline & & \multicolumn{3}{|c|}{$\mathrm{KM}$} & \multicolumn{3}{|c|}{ HEMASTIX } & \multicolumn{3}{|c|}{$\mathrm{KM}$} & \multicolumn{3}{|c|}{ HEMASTIX } \\
\hline & & B & $\mathrm{M}$ & $\mathrm{F}$ & $\mathrm{B}$ & $\mathrm{M}$ & $\mathrm{F}$ & B & $\mathrm{M}$ & $\mathrm{F}$ & $\mathrm{B}$ & $\mathrm{M}$ & $\mathrm{F}$ \\
\hline \multirow{5}{*}{$1: 4$} & 1 & + & + & + & + & + & + & + & + & + & + & + & + \\
\hline & 2 & + & + & + & + & + & + & + & + & + & + & + & + \\
\hline & 3 & + & + & + & + & + & + & + & + & + & + & + & + \\
\hline & 4 & + & + & + & + & + & + & + & + & + & + & + & + \\
\hline & 5 & + & + & + & + & + & + & + & + & + & + & + & + \\
\hline \multirow{5}{*}{$1: 16$} & 1 & + & + & + & + & + & + & + & + & + & + & + & + \\
\hline & 2 & + & + & + & + & + & + & + & + & + & + & + & + \\
\hline & 3 & + & + & - & + & + & + & + & + & + & + & + & + \\
\hline & 4 & + & + & + & + & + & + & + & + & + & + & + & + \\
\hline & 5 & + & + & - & + & + & + & + & + & + & + & + & + \\
\hline \multirow{5}{*}{$1: 64$} & 1 & - & - & - & + & + & + & + & + & + & + & + & + \\
\hline & 2 & - & - & - & + & + & + & + & + & + & + & + & + \\
\hline & 3 & - & - & + & + & + & + & + & + & + & + & + & + \\
\hline & 4 & - & - & - & + & + & + & + & + & + & + & + & + \\
\hline & 5 & - & - & - & + & + & + & + & + & + & + & + & + \\
\hline
\end{tabular}

Looking at the Hemastix results, no significant differences were found among species on the two types of material $(\mathrm{p}=1)$ and consistently positive results were observed for both materials.

For the KM results, there were no significant differences between species at dilutions 1:4 and 1:16 on fabric. At dilution 1:16, fish blood gave positive responses with KM only $60 \%$ of the time compared to the other species, but this difference was not statistically significant $(p=0,444)$. At dilution 1:64, KM gave a positive reaction to fish blood $20 \%$ of the time and no positive responses with the other two species; this difference was not statistically significant $(p=1)$.

On metal, no significant differences were found between the three species as both reagents gave positive responses $100 \%$ of the time. 


\subsection{Specificity}

When testing for false positives, Hemastix showed a much lower specificity than KM: tomato, potato, avian uric acid, bleach and sink rot consistently produced a colour change, and rust reacted $20 \%$ of the time. Interestingly, a KM reaction was absent for all substances in the absence of blood, except potato, which produced a colour change $60 \%$ of the time. Bleach alone did not react to KM, however, a mixture of blood and bleach produced a pink colouration at the addition of phenolphthalin alone, before hydrogen peroxide was added.

Since previous literature recorded false positive reactions of KM reagents over a longer time period, the time a colour change was seen was recorded for some substances (Table 4). All colour changes reported showed a strong pink colouration.

Table 4 - Substances reaction time

\begin{tabular}{|l|l|}
\hline Substance & Time \\
\hline Alsever's solution & $3 \mathrm{~m} \mathrm{08s}$ \\
\hline Bleach & Yink at 9m 26s \\
\hline Gun oil & $9 \mathrm{~m} 15 \mathrm{~s}$ \\
\hline Rust & $2 \mathrm{~m} 34 \mathrm{~s}$ \\
\hline Tomato & $1 \mathrm{~m} 43 \mathrm{~s}$ \\
\hline Sink rot & $56 \mathrm{~s}$ \\
\hline Bird urine & $58 \mathrm{~s}$ \\
\hline Chemgene & $2 \mathrm{~m} 27 \mathrm{~s}$ \\
\hline EDTA & No reaction after $10 \mathrm{~m}$ \\
\hline
\end{tabular}

For the species specificity experiment, all species gave positive results for all replicates and both reagents. 


\subsection{Stability}

Both reagents performed equally well under all four conditions tested for mammal and bird blood. However, KM performed worse than Hemastix on fish blood. Overall, Hemastix demonstrated a more consistent detection of aged blood than KM.

Except for the outside + sun strip, the stains on the other three strips gave positive results with both KM and Hemastix. The only notable difference on these three strips was the poorer performance of fish blood, which consistently gave more negative results with KM. Specifically, on the control strip fish blood gave positive responses $80 \%$ of the time using KM, against $100 \%$ using Hemastix (n.s., $\mathrm{p}=0,2241$ ); on inside + sun strip, fish blood treated with $\mathrm{KM}$ gave positive responses $40 \%$ of the time, against 100\% using Hemastix ( significant, $\mathrm{p}=0,0003$ ) and On outside + cover strip, fish blood treated with KM gave positive responses $26,7 \%$ of the time, against $100 \%$ using Hemastix (significant, $\mathrm{p}<0,0001$ )

However, for the outside + sun condition, only the mammal aged blood gave positive results with the two reagents. Significant differences were found between mammal blood and the other two species $(\mathrm{p}<0,00001)$ for both reagents under this condition. Mammal blood gave positive results $86,7 \%$ of the time with KM and $100 \%$ of the time with Hemastix; this difference was not statistically significant $(\mathrm{p}=0,48)$.

Comparing the different environmental conditions, fish blood consistently gave a larger number of negative results than the other two species when using KM: there is a significant difference in responses between both the inside + sun $(p=0,0341)$ or the outside + cover $(p=0,0092)$ and the control strip for fish blood. No difference was seen between the other two species.

Both bird and fish blood gave no positive results in the outside + sun scenario with both reagents, exhibiting a significant difference from the control strip for both reagents ( $p<0,00001)$. Mammal blood did not show significant differences in responses with either $\mathrm{KM}(\mathrm{p}=0,48)$ or Hemastix ( $p$ $=1$ ) under any condition.

\subsection{Effect on downstream DNA analysis}

The first swabs to be extracted were the 1:16 swabs treated with KM and 1:64 swabs treated with Hemastix for all three species, as these were the smallest dilutions to yield positive presumptive blood test results with the rub test (Table 2). The 1:64 was also the lowest dilution of blood showing stain coloration. 
For all three species, swabs treated with KM did not yield enough DNA for further analysis at dilutions 1:16, whereas swabs treated with Hemastix at the 1:64 dilution gave enough DNA for species identification. These results mean that the limit for reliable DNA extraction for swab treated with KM for all three species must be at a stronger blood dilution. Finally, visible stains treated with Hemastix have been demonstrated not to inhibit downstream DNA analysis as all extractions were successfully amplified by PCR.

\section{Discussion}

The validation experiments have assessed the sensitivity, specificity and stability of both presumptive blood test methodologies for use with mammal, bird and fish blood. While the results demonstrate that either test can be used to indicate the presence animal blood in a wildlife crime investigation, there are pros and cons to each method.

Of note is the great variability of both results and methodological application in the literature. Most importantly, there is no standardised threshold to discriminate between a positive and a negative from the KM reagent. Tobe et al. [1] chose a threshold of 4 minutes, whereas Webb et al. [5] chose a threshold of 10 seconds after the addition of hydrogen peroxide. During this project, a threshold of 30 seconds was chosen for KM testing in order to record results from diluted blood spots, while respecting the threshold for a reliable analysis.

The results of the direct testing in the first sensitivity study show the great sensitivity of both the KM and Hemastix. However, bird blood treated with KM gave positive results only down to dilution 1:256 (with a single exception at dilution1:1024). This may be explained if the original supply of bird blood had higher degree of dilution, since the supplier could not provide the exact concentration of blood in Alsever's solution. However, the results obtained with the Hemastix tests with bird blood were comparable with those obtained with the other two species.

A methodological discrepancy arises while using KM and Hemastix with the direct testing, which weakens the comparison of these results. While the KM reagents were spotted onto the stain and were therefore in direct contact with traces of blood on the stain, the Hemastix pad was placed in contact with the stain only for an instant. This may therefore account for the reduced sensitivity found using Hemastix during direct testing. Direct testing with Hemastix may be useful in real crime scene scenarios. However, placing the moistened pad of the Hemastix in contact with a stain is likely to result in the transfer of chemicals from the pad onto the stained surface. During direct testing, the stained filtered paper was observed to turn green almost instantaneously (less than 3 
seconds), even when a positive result on the Hemastix pad was recorded only after more than 20 seconds.

Using the rub-test, Webb et a. [5] found a sensitivity of 1:100 using both KM and Hemastix. . During this project, positive results from KM using the rub test were seen down to a dilution of $1: 16$, while positive reactions from Hemastix were observed down to a dilution of 1:64. In both sensitivity tests using the rub-test method, Hemastix performed better then KM detecting the presence of blood in a dilution of blood and water at one order of magnitude greater than KM reagents on porous materials, such as filter paper and fabric.

The better performance of the KM reagent on the metal surface (Table 3), compared to the first rubtest sensitivity study using filter paper (Table 2), could be explained by the lack of absorption of the stain by the material. The high porosity of fabric or filter paper is likely to reduce the amount of stain that can be sampled with a moistened swab when compared to a non-porous surface like metal.

The results of the first specificity test showed that, in absence of blood, Hemastix demonstrated broader reactivity with non-blood substances than KM. KM showed a false positive reaction in absence of blood only when testing potato. Contrary to Tobe et al. [1],bleach did not react with KM in the absence of the animal blood tested.

When dealing with the specificity of the reagents, it is important to consider that other studies have adopted different methodologies. Tobe at al. [1] chose to record any positive reaction within 4 minutes, while Cox [6] chose to record colour changes only at 20 and 60 seconds. This lack of consistency among studies inevitably gives rise to discrepancy with our results using the time thresholds of 30 seconds and 60 seconds, for KM and Hemastix respectively.

From the species specificity study, both reagents gave positive results all of the time with all species of animal blood tested. This demonstrates that both KM and Hemastix work as presumptive blood tests with a selection of vertebrate species across the evolutionary hierarchy that might commonly be encountered in wildlife forensic casework. Moreover, it demonstrates that the chemical reaction underpinning the two tests works with all sources of haemoglobin tested so far.

The stability tests showed both reagents to perform equivalently with both mammal and bird blood for the control, outside + covered and inside + sun strips, giving consistent positive reactions. Interestingly, KM was consistently less effective than Hemastix with fish blood. 
From the outside + sun test condition, only mammal blood was detected by both reagents, with no significant difference between the two. This result might indicate that mammal blood is more resistant to degradation. Another possible explanation might be the fact that mammal blood was the only type of blood to be stored neat, while blood from the other two species was preserved in Alsever's solution. This might have had an effect on the clotting capability of the different blood samples used.

The DNA analysis demonstrated KM to have a much greater inhibitory effect than Hemastix. This is in accordance with the literature that suggests that KM reagents reduce the possibility of extracting high-molecular-weight DNA [14]. Sloots and collegues [12] tested each component of the phenolphthalin reagent and demonstrated that sodium hydroxide was the likely cause of inhibition of DNA detection. The results from our exploratory DNA analysis suggest that swabs used to sample a visible blood stain - which was found to be 1:64 dilution in this study- from any species and then tested with Hemastix can be used for subsequent DNA analysis.

\section{Conclusion}

This study has provided an investigation of the strengths and weaknesses of two presumptive blood test methods used in forensic casework. Hemastix was demonstrated to be better suited for the needs of forensic wildlife casework, exhibiting higher sensitivity for rub-testing and offering less interference with DNA analysis, allowing the possibility of using a single swab for both blood presumptive testing and DNA extraction. Hemastix was found to be far less specific than KM with a number of household substances. However, the presumptive blood test is often only the first stage of analysis in a wildlife crime investigation, used to indicate possible sampling areas for DNA testing, and any positive result would be confirmed using DNA analysis to identify the species. Therefore, as false-positive Hemastix results will not impact on reporting - as no animal DNA will be produced from these false-positive results -the higher sensitivity of Hemastix makes it more appropriate for wildlife crime investigations. 


\section{Funding}

This research did not receive any specific grant from funding agencies in the public, commercial, or not-for-profit sectors.

\section{Author contributions}

Fabio Casali: Investigation, Formal Analysis, Writing-Original Draft. Sherryn A. Ciavaglia:

Methodology, Writing-Review \& Editing, Supervision. Chris Gannicliffe: Methodology, Writing-

Review \& Editing. Nathan Lidstone: Validation. Lucy M I Webster: Coneptualization,

Supervision, Writing-Review \& Editing.

\section{References}

[1] S. S. Tobe, N. Watson, and N. N. Daéid, "Evaluation of six presumptive tests for blood, their specificity, sensitivity, and effect on high molecular-weight DNA," J. Forensic Sci., vol. 52, no. 1, pp. 102-109, 2007.

[2] L. Teichmann, "Über die krystallisation des organischen bestandteile des blutes," Z Ration Med., vol. 3, pp. 375-388, 1853.

[3] Takayama, "No Title," Japanese J. Toxicol., 1912.

[4] M. Vennemann, G. Scott, L. Curran, F. Bittner, and S. S. Tobe, "Sensitivity and specificity of presumptive tests for blood, saliva and semen," Forensic Sci. Med. Pathol., vol. 10, no. 1, pp. 69-75, 2014.

[5] J. L. Webb, J. I. Creamer, and T. I. Quickenden, "A comparison of the presumptive luminol test for blood with four non-chemiluminescent forensic techniques," Luminescence, vol. 21, no. 4, pp. 214-220, 2006.

[6] M. Cox, "A study of the sensitivity and specificity of four presumptive tests for blood.," $J$. Forensic Sci., vol. 36, no. 5, pp. 1503-11, 1991.

[7] R. Winchester and H. Wansbrough, "Blood detection by chemical methods," XII-Biotech-ABlood Detect., pp. 1-6, 2013.

[8] L. Bayer HealthCare, "Hemastix Reagent Strips," 2004.

[9] J. E. Huffman and J. R. Wallace, "Species Identification," in Wildlife Forensics. Methods and Applications, 2012, pp. 273-275.

[10] H. Poon, J. Elliott, J. Modler, and C. Frégeau, "The use of Hemastix ${ }^{\circledR}$ and the subsequent lack of DNA recovery using the promega DNA IQTM system," J. Forensic Sci., vol. 54, no. 6, pp. 1278-1286, 2009.

[11] N. S. Alenazy, A. M. Refaat, and S. R. Babu, "Comparison of the effects of two presumptive test reagents on the ability to obtain STR profiles from minute bloodstains," Egypt. J. Forensic Sci., vol. 5, no. 3, pp. 103-108, 2015.

[12] J. Sloots, W. Lalonde, B. Reid, and J. Millman, "Kastle-Meyer blood test reagents are deleterious to DNA," Forensic Sci. Int., vol. 281, pp. 141-146, 2017. 
[13] J. H. McDonald, Handbook of Biological Statistics (3rd ed.). Baltimore, Maryland: Sparky House Publishing, 2014.

[14] M. N. Hochmeister, B. Budowle, and F. Samuel Baechtel, Effects of presumptive test reagents on the ability to obtain restriction fragment length polymorphism (RFLP) patterns from blood and semen stains, vol. 36. 1991. 